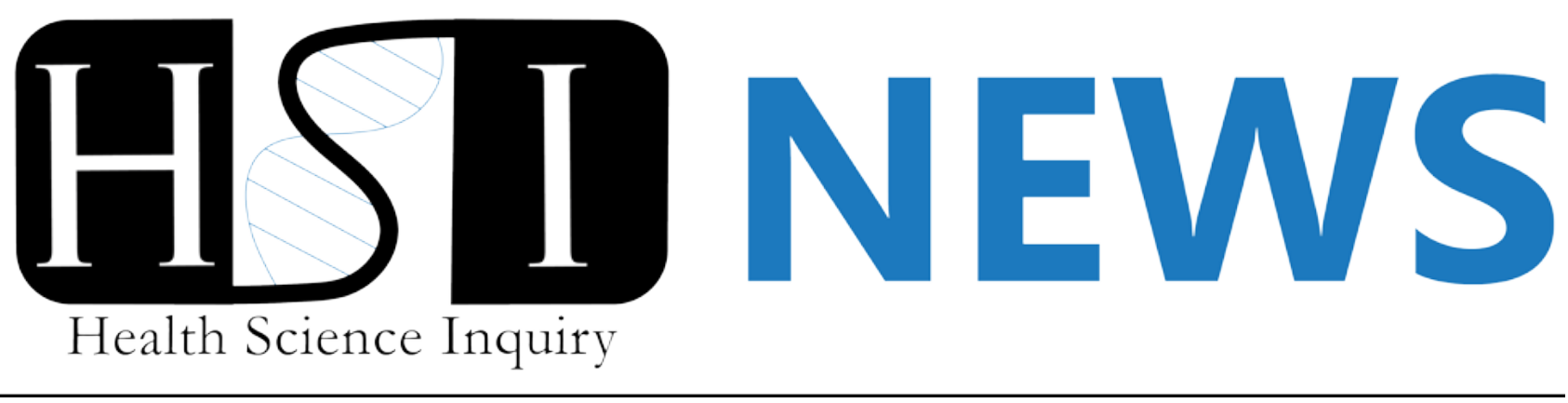

\title{
The best way forward: simulations and machine learning for optimizing treatment
}

-Kevin Dick

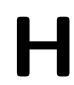
appening upon a bifurcation in a road, one is faced with a simple choice: left or right? Without complete information about the paths ahead, this can present a difficult choice. Depending on the context of your journey and the information and knowledge you have, you make an informed decision. In different situations, that decision might be more complex: left, right, go back, stay here... At times it is beneficial to learn more about the paths ahead before committing to one, thereby improving the expected outcome of your final decision. This is an oversimplification of the decision process we as humans perform every day in all situations, and something that Dr. Audrey Durand has used to make quantitatively informed decisions for a plethora of health conditions. As a post-doctoral researcher at the McGill University School of Computer Science, she has helped guide the decision-making process in research on Down syndrome, osteoporosis, heart disease, cancer, and Alzheimer's disease. In an interview with Dr. Durand, we garnered some insights into the state-of-the-art machine learning methods in health care.

\section{Simulations for the Screening of Fetal Down Syndrome}

Dr. Durand's first use of computer science in the domain of health care began with the development of simulators to evaluate Down syndrome screening strategies during pregnancy. While prenatal foetus karyotyping is considered the gold standard for the detection of Down syndrome, the use of reliable molecular methods to detect common aneuploidies (the presence of an abnormal number of chromosomes) are considered faster and less expensive. One such method is rapid aneuploidy diagnosis (RAD), which includes techniques such as fluorescence in situ hybridization (FISH) and quantitative fluorescence PCR (QF-PCR). To determine the optimal screening options trading-off various outcomes of Down syndrome prenatal screening such as cost, false positive rate, and number of procedure-related miscarriages, Dr. Durand and collaborators performed a series of computer simulations. These comprised estimating the performance of six screening options recommended by guidelines in the US and Canada, combined with three diagnostic approaches (karyotyping, FISH or QF-PCR) for each end point that cover the main outcomes in Down syndrome prenatal screening. Their work indicated that RAD detected the majority of clinically significant chromosomal abnormalities while remaining the most cost-effective option [1]. These results promise to partially offset the economic and financial burden on contemporary medical systems. Following these successes, Dr. Durand went on to adopt state-of-the-art methods to address similar optimization challenges.

\section{Adaptive Clinical Trials as Bandit Problems}

In machine learning, researchers want to teach a computer (known as an "agent") to "understand" a problem and then use it to make subsequent decisions. Dr. Durand's expertise in machine learning (ML) specializes on navigating the exploration-exploitation trade-off. This arises when learning about the problem is conducted simultaneously as the agent is making decisions. Roughly akin to the path-finding example above, Dr. Durand explains: 
determining the optimal behaviour, and behaving in what is thought to be the 'best' way given current knowledge. In machine learning, this is known as 'bandits problems'."

Dr. Durand went on to explain that she is currently involved in a project where bandits algorithms are used to guide an adaptive clinical trial in real mice experiments with induced cancer tumours. Given that personalized medicine seeks to customize treatment based on an individual patient's characteristics, the pharmacological treatment strategy can be modelled as a contextual bandit problem which trades-off the collection of information about the treatment believed to be optimal given the current state of the patient and the exploration of lesser known treatments that could improve the current strategy. The pilot project applying this strategy over simulations, in combination with preliminary results from mouse model experiments, showed considerable promise [2], and the subsequent analysis and dissemination of results are forthcoming and highly anticipated.

\section{A New Age of Medicine}

In summary, path finding in the face of incomplete information is a challenge ubiquitous in healthcare research as it is in the navigation of our own natural environments. This research looks to quantitatively improve healthcare treatment while remaining cost-effective. Through both her research and vision for the future of healthcare, Dr. Durand is helping to herald a new age of medicine:

"With machine learning, resources can be used more efficiently, meaning that we can save time, money, and more importantly, lives. By automating some procedures, machine learning can even provide some populations with access to services that were otherwise not available. Healthcare is a field that will highly benefit from machine learning."

\section{References}

[1] Gekas, J., van den Berg, D.-G., Durand, A., Vallée, M., Wildschut, H.I.J., Bujold, E., et al. (2011) Rapid testing versus karyotyping in Downs syndrome screening: cost-effectiveness and detection of clinically significant chromosome abnormalities. Eur J Hum Genet, Available from: http://www.ncbi.nlm.nih.gov/pubmed/20842178.

[2] Durand, A., Pineau, J. (2015) Adaptive Treatment Allocation Using Sub-Sampled Gaussian Processes. In: 2015 AAAI Fall Symposium Series.

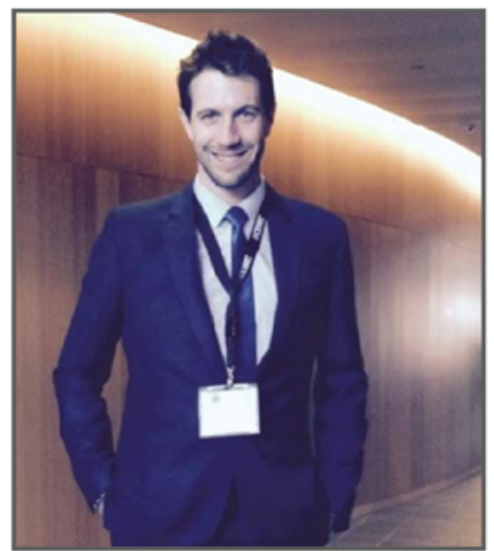

Kevin is a third year $\mathrm{PhD}$ candidate in Biomedical Engineering specializing in Data Science and Bioinformatics at Carleton University. He received his BSc in Biology and Computer Science from McGill University in 2014. His doctoral research focuses on improving protein-protein interaction prediction and leveraging context in related complete-graph problems such as protein function prediction and medical diagnosis prediction. As a polymath, Kevin spends his spare time exploring his curiosities at the intersection of the arts and sciences. 